\section{Do doctors have to have the covid-19 vaccine?}

\section{Abi Rimmer asks four experts whether doctors must be vaccinated against covid-19}

\section{Abi Rimmer}

\section{We have a duty to protect patients}

Vageesh Jain, public health specialty registrar, University College London, says, "As it stands, legally, you don't have to have a covid vaccine. But ethically, clinically, epidemiologically-whichever way you slice it-I would argue you do.

"The ethical case is grounded in the professional duty healthcare workers have to protect their patients: do no harm. Although everyone has the right to decide whether to take a vaccine or not, patients (often elderly or unwell, and therefore vulnerable to covid-19) also have fundamental rights to be protected from avoidable harm. When these principles come into conflict, actions that safeguard the best interests of patients must be favoured.

"The evidence on covid-19 vaccines is clear: they are safe and they work. Some are understandably concerned about a lack of long term data, but the importance of this can be overstated. For other vaccines, mild side effects can occur in the first few months but serious and longer term problems are largely theoretical for most. On the other hand, the clinical risks of covid-19 (including long covid) are real, especially for those working in healthcare, where the chance of encountering an infectious disease is relatively high.

"Vaccines don't just protect you and your patients, but the wider population too. Hospitals and community practices are amplifiers of transmission: one or two cases can rapidly proliferate. Outbreaks in institutional settings must therefore be prevented. The best way to do this is through vaccination. This isn't just important for covid-19. When health facilities are hit by outbreaks, the disruption and reorganisation of local systems and public reluctance to seek care carries far reaching implications for health.

"In the UK, most healthcare workers offered a jab have rolled up their sleeves, but the rate of uptake in some groups, including ethnic minority staff, is low. There is now a legitimate debate taking place on whether to make vaccination mandatory for health workers. If the voluntary approach fails, more restrictive measures may be justified, in which case you would have to have a vaccine."

\section{We should lead by example}

Colin Melville, General Medical Council medical director and director of education and standards, says, "It's been an incredibly challenging year with many uncertainties and difficult decisions about patient care, alongside concerns about our own wellbeing and the wellbeing of those around us. The arrival of covid-19 vaccines provides new tools to protect patients, doctors, and their families, and with the progress of the rollout we are, hopefully, starting to see a light at the end of the tunnel.

"Most, if not all, doctors should now have been offered one of the vaccines. And, for most, the benefits are clear. But some may be hesitant, and we've received queries from doctors asking what our guidance says about being vaccinated.

"Our advice, set out in our guidance on the professional standards expected of all doctors, ${ }^{1}$ says, 'doctors should be immunised against common serious communicable diseases, unless this is contraindicated.'

"So, while there is no absolute duty to be vaccinated, you must consider the risk of spreading coronavirus to patients, particularly those who are clinically vulnerable. If you have good reason not to be vaccinated, you will need to take appropriate steps to reduce risks to patients and prioritise their safety. And if you're unsure, please seek appropriate clinical advice to support you in making the decision.

"Choosing to have the vaccine or not is a personal choice dependent on individual circumstances. But at a time when health professionals have never been more valued, we should lead by example.

"You can find other advice on helping patients to make informed decisions and information about the GMC's guidance on our ethical hub online."2

\section{If you refuse, prepare to justify why}

Rob Hendry, medical director at the Medical Protection Society, says, "While the vaccination is recommended, it's not currently mandatory and there are no contractual obligations, so healthcare workers cannot be forced to have it.

“They should, however, be mindful of the GMC's Good Medical Practice, which states that 'doctors should be immunised against common serious communicable diseases, unless this is contraindicated.' Its covid-19 guidance also states that 'there is a potential risk of inadvertently spreading coronavirus to vulnerable patients. This weighs in favour of doctors being vaccinated unless there are good reasons why it isn't appropriate in your individual circumstances.'

"Medical staff who refuse the vaccine should therefore be prepared to justify their decision and, where possible, mitigate any risks. We would encourage healthcare workers who are reluctant or refuse the vaccine to explore their increased risk of repeated exposure to the virus, the benefits of the vaccination to colleagues, patients, and family, and the wider benefits of reducing the transmission of 
covid-19 with their supervisor and occupational health.

"If the reluctance relates to a health concern, forcing a healthcare worker to receive the vaccine could be seen as unlawful discrimination under the Equality Act 2010. If the refusal is because of religious beliefs, this should also be respected under employment laws.

"We would expect an employer to undertake a risk assessment of a healthcare worker who is unwilling or unable to have the vaccination, and consider how risks could be mitigated, such as redeployment to a non-patient facing role, or remote working.

"This would demonstrate what steps have been considered to balance the interests of the individual and others. Ultimately, the viability of any solution must focus on reducing risks and prioritising patient safety. NHS England has set out similar advice for employers with staff refusing the vaccination."

\section{We can protect others by being vaccinated}

Sarah Ali, consultant in diabetes and endocrinology, Royal Free London NHS Foundation Trust, says, "Working as a patient facing doctor and looking after people who are considered to be clinically vulnerable meant that taking the vaccine was an easy decision for me.

"Based on the premise that vaccines are effective at reducing the transmission of covid-19, I saw it as protecting not only myself but also the community, especially those at high risk of morbidity and mortality from covid-19 and those who are unable to have the vaccine.

"Additionally, I consider the vaccine to be our strongest tool to exit the pandemic in a timely manner, and minimise the significant economic, societal, and psychological impact the pandemic is continuing to have.

"I appreciate, however, that it would be challenging to make the vaccine mandatory for all NHS colleagues. People should be able to come to their own decision; forcing a vaccine on someone who is unsure or unwilling could lead to distrust.

"There are a small number of people who are vaccine hesitant. Resolving this will involve having humble, honest, and uncomfortable discussions as well as offering them education and support. Making vaccines mandatory inhibits the opportunity to have these open discussions and could antagonise people and promote distrust.

"Making the decision to have mandatory covid vaccines is therefore challenging and needs to be done with real considerations of both sides of the argument.”

1 General Medical Council. Good medical practice. 2019. www.gmc-uk.org/ethical-guidance/ethicalguidance-for-doctors/good-medical-practice.

2 General Medical Council. Coronavirus: your frequently asked questions. www.gmc-uk.org/ethicalguidance/ethical-hub/covid-19-questions-and-answers 